\title{
A multiobjective fleet location problem solved by adaptation of evolutionary algorithms NSGA-II and SMS-EMOA
}

\author{
Bir çokamaçlı filo konuşlandırma probleminin NSGA-II ve SMS-EMOA \\ evrimsel algoritmalarının uyarlanması ile çözümü
}

\author{
Ertan YAKICI ${ }^{*}$ iD \\ ${ }^{1}$ Industrial Engineering Department, Naval Academy, National Defense University, Istanbul, Turkey. \\ rtnykc@gmail.com
}

Received/Geliș Tarihi: 09.11.2016, Accepted/Kabul Tarihi: 12.06.2017

* Corresponding author/Yazışılan Yazar

doi: $10.5505 /$ pajes.2017.20688 Research Article/Araștırma Makalesi

\begin{abstract}
The problem of locating naval platforms in the operation region with the aim of maximizing both total radar coverage and critical radar coverage is solved by using Multiobjective Evolutionary Algorithms (MOEA). Non-Dominated Sorting Genetic Algorithm-II (NSGA-II) and $S$-Metric Selection Evolutionary Multiobjective Optimization Algorithm (SMS-EMOA) procedures are implemented. Experiments show that evolutionary algorithms provide good and diverse alternatives that are considered to be very close to Pareto-optimal front. The performances of NSGA-II and SMS-EMOA approaches are compared employing the hypervolume indicator technique. The performance of NSGA-II is found better in terms of both convergence and diversity.
\end{abstract}

Keywords: Fleet location, Optimal sensor placement, Multiobjective evolutionary algorithms

\section{Introduction}

Usage of fixed sensors as surveillance and reconnaissance assets is a common practice in military operations. Any stationary sensor, such as radar installations or naval platforms not in motion can be used to provide situational awareness in a certain area. These fixed sensors are placed at predetermined locations but may be moved between uses [1].

In this study, the problem involves optimization of locating sensor carrying units with the restrictions caused by terrain's geographical structure. The objective is maximizing both of total sensor coverage and critical region sensor coverage. The problem is motivated by the requirement of establishing and maintaining the surface picture just before the possible engagements start in a naval battle space which has several lands that prevent radar emmisions to reach their utmost extent.

The problem of determining an optimal configuration of sensors is a challenging problem when there are several constraints related to geographical terrain and the structure of configuration itself, even when the available number of these sensors and their ranges are determined and given as parameters of the problem.

The scope of this study is adapting and applying a widely known Multiobjective Evolutionary Algorithm (MOEA), NonDominated Sorting Genetic Algorithm (NSGA-II) [2], and a relatively new approach, S-Metric Selection Evolutionary Multiobjective Optimization Algorithm (SMS-EMOA) [3], on the two-objective sensor coverage problem in order to provide
Öz

Donanma platformlarının, radar toplam kapsama alanları ve radar kritik kapsama alanlarının ençoklanması amacı ile harekât bölgesinde konuşlandırılması problemi çok amaçlı evrimsel algoritmalar kullanılarak çözülmüstür. Bu kapsamda, literatürde Non-Dominated Sorting Genetic Algorithm-II (NSGA-II) ve S-Metric Selection Evolutionary Multiobjective Optimization Algorithm (SMS-EMOA) adl verilen yöntemler kullanılmıștır. Deney uygulamasında, bu yöntemlerin Pareto-optimal cepheye oldukça yakın olduğu değerlendirilen iyi ve istendiği gibi birbirinden farklı çözümler ürettiği görülmüştür. Kullanılan yöntemlerin performanslarl hipervolüm gösterge tekniği kullanılarak karșılaștırılmıș, NSGA-II yönteminin daha iyi performans gösterdiği tespit edilmiştir.

Anahtar kelimeler: Filo konumlandırma, Optimal sensör yerleşimi, Cok amaçlı evrimsel algoritmalar

decision maker with several non-dominated solutions close to true Pareto-optimal front. We also compare these two approaches for our problem.

Computational intelligence methods like genetic algorithms, multiobjective genetic algorithms, swarm intelligence, and operations research techniques has been widely used in the analyses of sensor networks. These analyses include determining sensor placements, number and type of sensors, optimization of power usage, clustering, network topology and routing [1]. Using optimization techniques to determine sensor placement can improve the effectiveness of the sensor suites. In the problems found in relevant literature, different objectives are considered for different types of sensors, like maximizing the probability of detection, minimizing the time to detect, maximizing the coverage of the sensors, etc. While some studies use multiobjective optimization methods to find a set of non-dominated solutions [4], [5], others use weights to have a combined objective function [6], [7]. Most of the studies in the literature address wireless sensors which have limited lifetime. Therefore, most of them consider the tradeoffs between coverage and lifetime. A broad review of multiobjective optimization in wireless sensor networks is recently given by Fei et al. [8].

It has been shown by previous works that MOEA approaches can successfully address the problem of optimal organization and formation of teams of sensors. Bugajska and Schultz [9] study the co-evolution of the form and function for autonomous agents. Characteristics that can be evolved are the number of sensors in a sensor suite and the coverage area 
of each sensor. The possible locations for the sensors are kept fixed and the maximum number of sensors is limited. In another study [10], they add a new characteristic: the detection angle and the placement of the sensors.

An algorithm is presented by Chaudhry et al. [11] that uses an MOEA approach to solve sensor placement optimization problem when the number of sensor nodes is not fixed and the limit on the number of nodes is not given.

Martins et al. [12] propose a multiobjective hybrid optimization algorithm for solving a sensor coverage and connectivity problem. They use a multiobjective genetic algorithm to improve the solution. Khalesian and Delavar [13] propose a constrained MOEA to maximize the sensor coverage and minimize energy consumption while maintaining connectivity. Another recent study by Jameii et al. [14] consider conflicting objectives of lifetime, coverage and connectivity simultaneously and they employ NSGA-II to solve the problem.

Sakr and Wesolkowski [4] use multiobjective genetic algorithm to maximize total coverage, minimize total cost and minimize coverage overlap, assuming a fixed number of sensors.

Sengupta et al. [15] propose a multiobjective optimization algorithm for scheduling nodes of a sensor network while maximizing lifetime. They focus on the probabilistic coverage on regions with different level of sensing requirements. The algorithm attemps to find a good tradeoff among coverage, lifetime and energy consumption. In another study, Sengupta et al. [16] utilize the same algorithm in solving another problem which has one more additional objective of minimizing the number of deployed sensor nodes.

Bara'a et al. [17] use NSGA-II to solve the problem which integrates the coverage maximization of mobile sensor networks with efficient routing that maximizes network lifetime.

In the context of military situational awareness, Ball and Wesolkowski [18] apply NSGA-II to determine the sensor locations with the objectives of maximizing number of detected vehicles, minimizing number of coverage discontinuities and minimizing network cost.

The problem which is closely related to ours is proposed by Küçükali [19]. The author applies a single objective genetic algorithm in order to maximize sensor coverage without sacrificing the strategic zones. He worked on a very similar case as the one studied in this work, but with more assumptions and restrictions on certain parameters. In his work, two objectives are linearly combined into a single objective.

To the best of our knowledge, no study using multiobjective methods is reported before in the literature, which analyzes the problem of maximization of total and critical coverage in an environment where these two objectives conflict. In this sense, the contribution of this study is application of two evolutionary multiobjective solution methods to an interesting problem which is not studied except once [19], and comparison of their performances.

Next section outlines the problem environment and explains the applied algorithms. Sections 3 and 4 present the findings related to our experiment and the conclusion of this study, respectively.

\section{Problem statement and solution methods}

In this section, the problem and two different evolutionary methods employed for solving our problem are explained.

\subsection{Problem definition and decision space}

The problem environment is a naval battlespace with several islands which can be considered as two-dimensional plane with obstacles. Since all of the radar carrying platforms are ships and emissions of these radars cannot go over the obstacles, a point that is behind an obstacle cannot be covered. In this environment, some regions like critical straits, naval bases or harbors are of more importance. Since these regions are very close or adjacent to the lands, two objectives of the problem, maximization of total coverage and maximization of critical coverage, have conflict with each other.

The sensor carrying units have to be partitioned into smaller groups that form the fleets and each fleet have its own flagship which serves as the tactical commander of that fleet. Moreover, because of tactical warfare requirements, each ship must remain in a predetermined range of the flagship of the fleet to which it is assigned. While the flagships remain unchanged, the fleets can exchange their members which may have different range of sensors. Since the number of fleets is not that flexible in the formation of a naval force, the algorithm is not allowed to evolve on this characteristic.

The decision space is a digital image file which represents the map of a possible operation region. In this map, sea, land and the critical region at sea are represented by certain different colors for identification of the pixels. The location of each unit is encoded as coordinate values. Although it is unlikely, as there is no restriction, multiple units may have the same location. The probability of this case to occur increases as the islands forms a circle-like shape around a fleet and/or the allowed maximum distance between flagships and the other ships decreases. Even if this case occurs, it may be considered as a feasible solution as long as the real size of a pixel allow the ships to have same coordinates. Any infeasible solution that might be generated, like locating a unit on land, is blocked in the implementation of the algorithms. Since all of our constraints are considered to be hard and avoided, the constraint handling techniques of MOEA are not employed.

\subsection{Solution representation}

Each candidate solution in the population specifies the arrangement of the given set of units encoded as chromosomes formed by genes. Each gene representing a fleet keeps evolvable coordinate values ( $x$ and $y$ ) of the flagship ( $f$ ) and the other ships in that fleet. Another parameter, range, indicates the sensor range associated with the corresponding ship.

An example for a chromosome representing an individual in the population is shown in Figure 1. The solution in this example have $M$ fleets in total, $n_{1}+1$ ships in the first fleet and $\mathrm{n}_{2}+1$ ships in the second fleet.

\begin{tabular}{|c|c|c|c|}
\hline Fleet 1 & Fleet 2 & $\ldots$ & Fleet M \\
\hline$x(1, f), y(1, f)$ & range $(1, f)$ & $x(2, \mathrm{f}), y(2, \mathrm{f})$ & range $(2, \mathrm{f})$ \\
\hline$x(1,1), y(1,1)$ & range $(1,1)$ & $x(2,1), y(2,1)$ & range $(2,1)$ \\
\hline$x(1,2), y(1,2)$ & range $(1,2)$ & $x(2,2), y(2,2)$ & range $(2,2)$ \\
\hline$\ldots$ & $\ldots$ & $\ldots$ & $\ldots$ \\
\hline$x\left(1, n_{1}\right), y\left(1, n_{1}\right)$ & range $\left(1, n_{1}\right)$ & $x\left(2, \mathrm{n}_{2}\right), y\left(2, \mathrm{n}_{2}\right)$ & range $\left(2, \mathrm{n}_{2}\right)$ \\
\hline
\end{tabular}

Figure 1: Representation of a solution. 


\subsection{Algorithms}

In applying NSGA-II (see Figure 2) which uses an explicit diversity-preserving mechanism for the goal of maintaining diversity on the obtained pseudo Pareto-optimal front, the individuals of the initial population are generated by random selection from the coordinates at sea. Objective values and crowding distance values of these initial chromosomes are calculated and assigned before the start of iterations. Crowding distance is a measure of the density of solutions surrounding a particular solution on a front. In our case which is a 2-objective problem, this measure is equal to $L_{1}$ distance between neighboring two solutions.

At each iteration, firstly, the mating pool is populated by crowded tournament selection operation [2]. The crowded tournament operator compares two solutions and returns the winner of the tournament. A solution wins a tournament with another solution, if it has a better non-domination rank, or if both have the same rank but it has a better crowding distance.

After filling up the mating pool, ensuring that each individual participated in the tournament for two times, the offspring are generated by crossover and mutation operations. First, a uniform crossover operation is applied to each member of the mating pool and then all of those members are mutated with respect to a uniform probability distribution. If a gene is selected for mutation, the coordinates of the flagship is assigned randomly. The other ships (escortships) of the fleet that is represented by the mutated gene are also assigned coordinates probabilistically with respect to a probability distribution function which linearly favors farther points within the specified distance interval of the flagship. This interval has an upper limit due to tactical conditions and a lower limit which is equal to the difference between sensor ranges of the considered flagship and escortship, assuming that flagship has greater sensor range.

After exploration operations are applied, objective values and crowding distances are assigned to new offspring. Nondominated sorting and crowding distance sorting in each nondominated front is applied to the merged population of parent and offspring chromosomes. The non-dominated sorting is used to classify the entire population consisting of parent and offspring solutions that is output of the last iteration. After the non-dominated sorting is over, the new parent population is formed by best non-dominated fronts. When the last allowed front is considered, the number of remaining slots may be less than the number of solutions on the front. This case, which is very likely to occur after a number of iterations, is handled by crowding distance sort method to choose the members of the last front [2]. This method chooses the solutions which reside in the least crowded region within non-dominated front. During the conversion of the population to the Pareto-optimal front, this algorithm ensures a better diversity among the individuals on the solution space. New generation is selected from the sorted list according to the allowed population size which is half of the merged population size in this algorithm. When the predetermined final iteration is reached, the algorithm generates the output of the first front solutions for presentation to decision maker.

Most of the computational cost is incurred in the computation of objective values. The coverage and critical coverage of each chromosome is calculated by union of the sets of pixels covered by each unit of that chromosome. For each unit, the pixels are checked for each line of sight originating from its coordinates until a point of land is encountered or maximum range of sensor is reached. The computational time, for each unit, increases proportional to the square of its sensor range. Therefore, the number of units, sensor ranges, the size of population and the layout of lands on the decision space collectively determine the computational time of the algorithm.

As an alternative approach, SMS-EMOA is applied. This approach combines the ideas from NSGA-II and archiving strategies presented by Knowles et al. [20] and Knowles and Corne [21]. This steady state algorithm ranks the solutions by non-dominated sorting. The hypervolume procedure is applied in order to discard the solution having the least hypervolume contribution to the worst non-dominated front [3]. At each iteration, a new solution generated by crossover and mutation operators, becomes a member of the population if this replacement with another individual is accepted with respect to the criteria given in the SMS-EMOA algorithm presented in Figure 3. After the application of non-dominated sorting, a reduction procedure is called in order to eliminate the worst individual [3]. In this procedure, the individual which is dominated by most of the other individuals is discarded. If there is only one front, S-metric (also known as hypervolume measure) is used [3]. Hypervolume (or hypervolume indicator $(\mathrm{HI})$ ) is a quality measure for a set $S$ consisting of solutions which do not dominate each other. In our case, which is a 2-objective maximization problem, this measure is the area of the region which is simultaneously dominated by the solutions in $S$ and bounded below by a reference point on two dimensional solution space.

\subsection{Evaluation of objective functions}

For a given coordinates of a unit, $(a, b)$, with a sensor range of $R$ NMs and a map width of $W$ NMs, total coverage and critical coverage of the unit is found by the algorithm presented as follows:

Let the length of an edge of a square pixel be $w\left(w=W / \max _{-} x\right.$, where $\max _{-} x$ is the width of the map in terms of pixels) and the radar range in terms of pixel edge length be $r$ (integer number obtained by rounding down the ratio $R / w$ ). Let the color attribute indicates whether a pixel is in noncritical sea region, or in critical sea region (red), or on land (green).

Step 1. : Construct $S$, the set of pixels those form the square which has pixel $(a, b)$ at its center and edge length of $2 r$. Set $C$ to 0 where $C$ represents the set of pixels covered by radar at pixel $(a, b)$.

Step 2. : Set $\left(a^{\prime}, b^{\prime}\right)$ as $(a, b)$. Choose any one of the pixels $(x, y)$ in set $S$ if there is any, o.w. terminate.

Step 3. : Choose the closest pixel, let say pixel $(z, w)$ among eight neighbor pixels of the pixel $\left(a^{\prime}, b^{\prime}\right)$ to the pixel $(x, y)$.

Step 4. : If $(z, w)$ is within the range of $r$ and at sea (determined by checking its color attribute), then $C:=C \mathrm{U}(z, w)$ and $\left(a^{\prime}, b^{\prime}\right):=(z, w)$ and go to step 3. Else $S:=S \backslash(x, y)$ and go to step 2 .

An example for objective function evaluation is illustrated in Figure 4. White circle, with radius equal to $r$, represents the boundary of coverable region with respect to radar range. Gray rectangle is the boundary of the set of pixels those form 
the square which has pixel $(a, b)$ at its center and edge length of $2 r$. Gray area around pixels $(a, b)$ and $(z, w)$ represents neighbor pixels.

The union of covered pixels by all of the units in the chromosome are counted and the area of this union which represents the first objective value is calculated in square NMs. The second objective function value is equal to the area of critical (red) pixels within the set of all covered pixels.

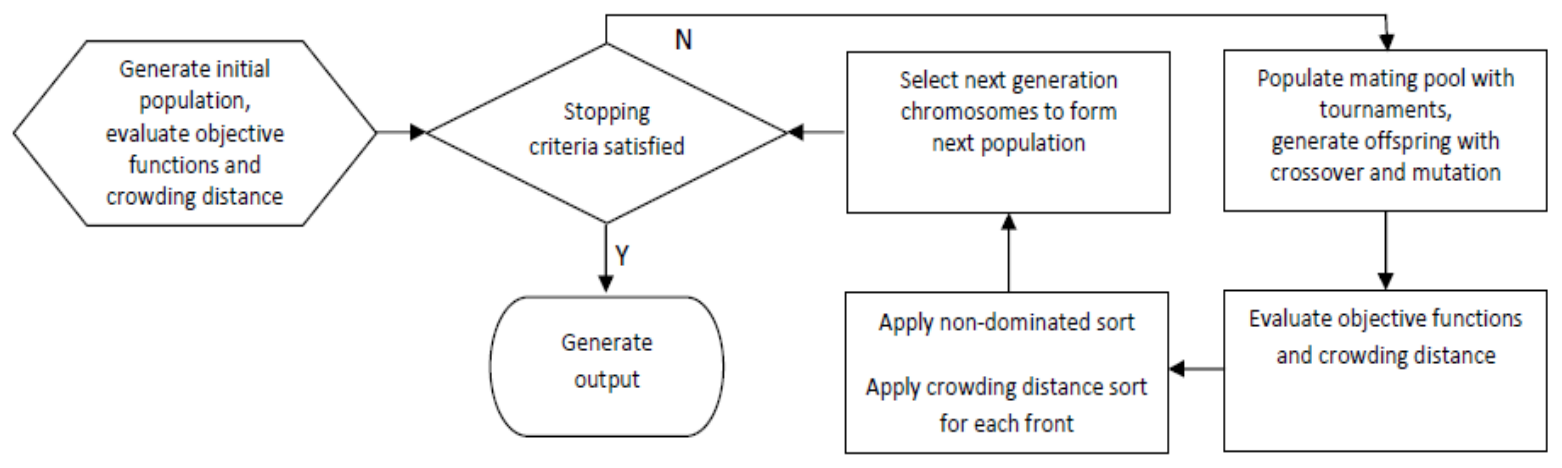

Figure 2: Flowchart of NSGA-II.

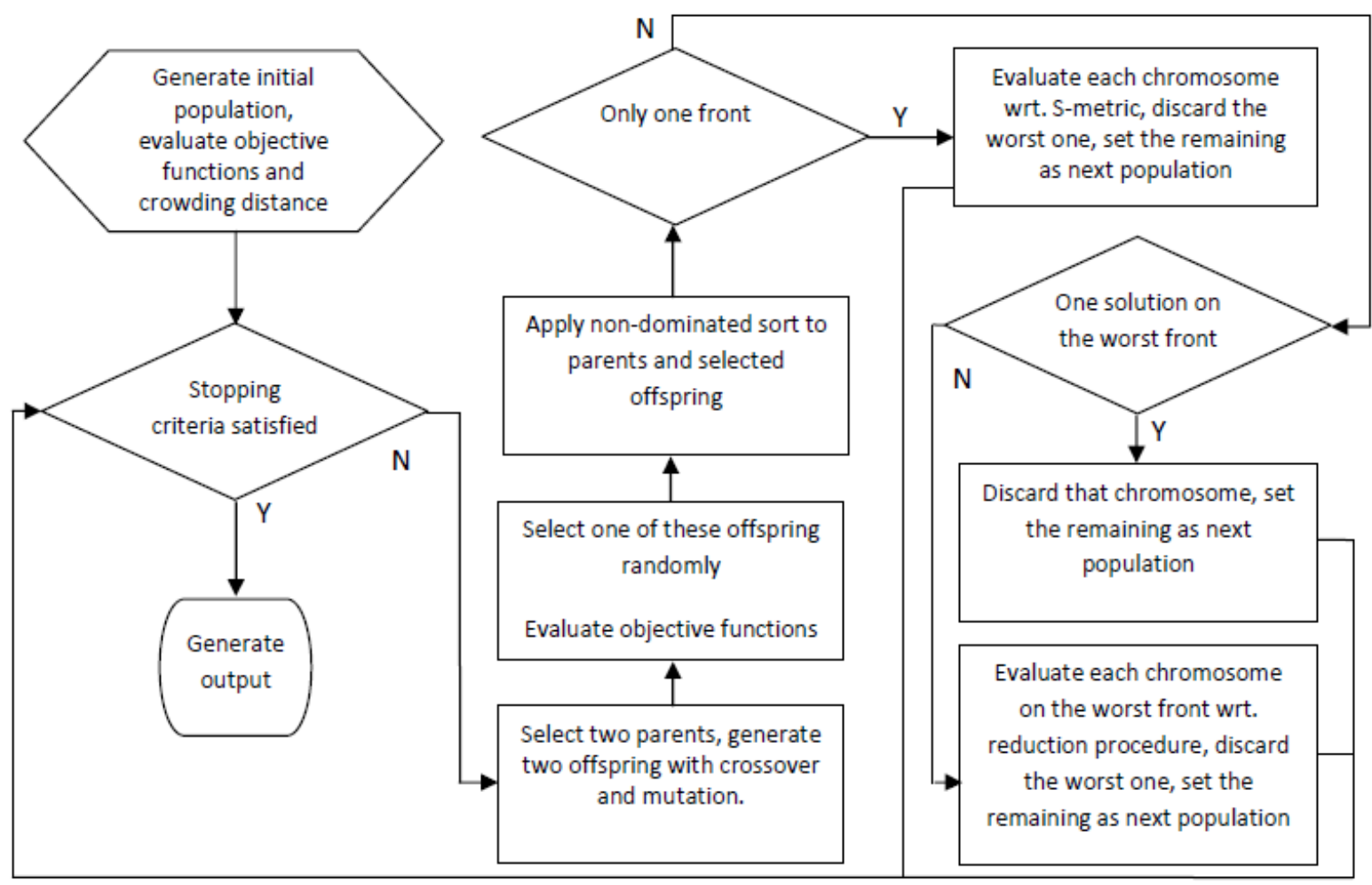

Figure 3: Flowchart of SMS-EMOA.

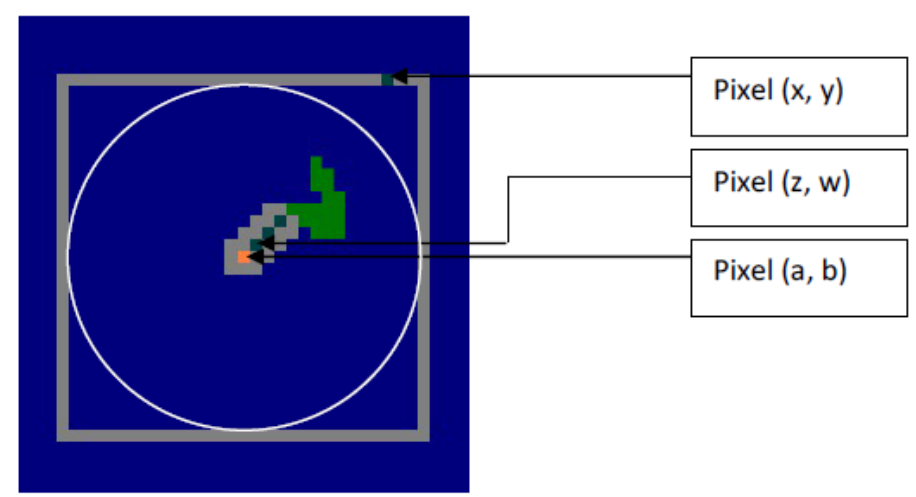

Figure 4: Illustration of objective function evaluation. 


\section{Experiment}

As a decision space, a map of Eastern Aegean Sea, presented in Figure 5, is used with assumptive critical regions close to the coast of Turkish main land. It is obvious that the performance of any algorithm must be strictly dependent on the decision space represented by a geographical structure and critical regions composed on it.

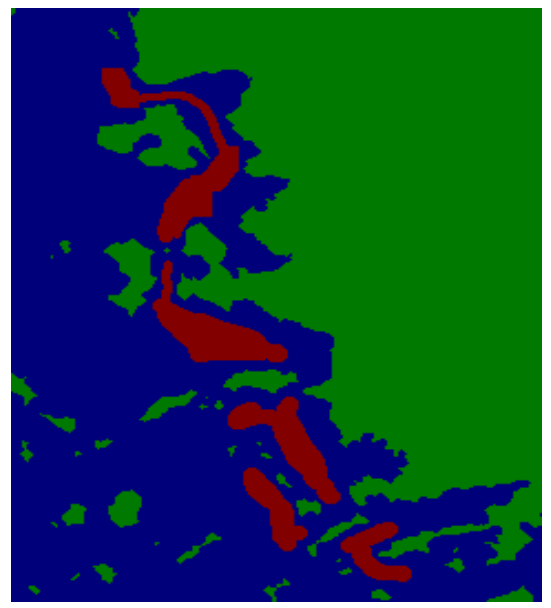

Figure 5: The map of Eastern Aegean Sea used in the experiment (real width=240 NM, $240 \times 327$ pixels).

Number of flagships and escortships (for each flagship), radar range for each of the units, the maximum distance between an escortship and the flagship to which it is assigned are the parameters specific to the problem. In the experiment, we set the number of flagships to 5 , and their sensor ranges to $25 \mathrm{NM}$. We assume that, 4 of the flagships need 2 escortships, while one of them needs 3 . The sensor ranges of 4 escortships out of 11 are set to $10 \mathrm{NM}$, while the sensor ranges of others are set to 7 NM. Maximum distance of an escortship to the flagship to which it is assigned is allowed to be $30 \mathrm{NM}$. A feasible solution to this problem instance is presented in Figure 6. In the maps given in Figures 5 and 6, non-critical sea region, critical sea region and land is painted in blue, red and yellow, respectively. In Figure 6, covered region in the solution is painted in gray, while the points within covered region represent the locations of units, where flagships are indicated by bigger points.

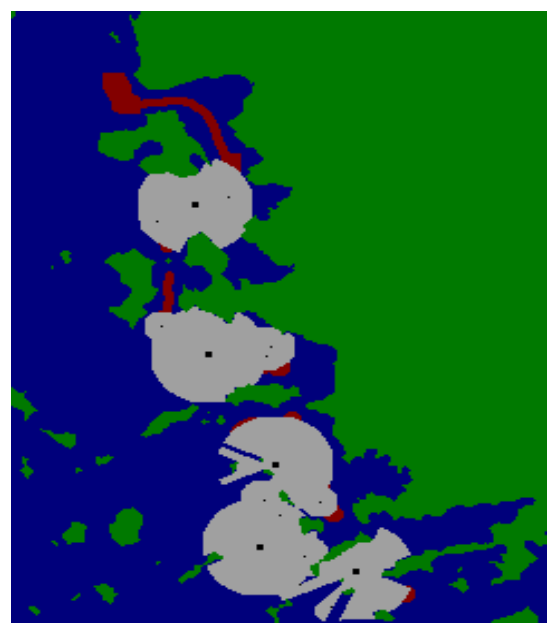

Figure 6: A feasible solution to the experimented problem instance.
30 runs with 100 iterations of NSGA-II and 30 runs with 1000 iterations of SMS-EMOA are executed. We set population size to 20 , crossover and mutation probability to 0.5 . In Figures 7 and 8, each plot represents the averages of population average values and population best values for two objective functions (f1: total coverage and f2: critical coverage) within each iteration for the first hundred iterations. These graphs give an idea about the convergence performance of two approaches.

In the experiments with NSGA-II, consistently most of the convergence observed at first iterations. As iteration number increases, as expected, convergence slows down and finding better solutions which are closer to the true Pareto-optimal front requires more and more iterations. This pattern is also valid for SMS-EMOA as seen on Figure 9. However since SMS-EMOA convergence rate is lower, much more iterations are needed in order to have convergence as much as that of NSGA-II attains in hundred iterations. On the other hand, since NSGA-II requires a number of offspring to be evaluated at each iteration, this algorithm is slower than SMS-EMOA approach. For the case studied, an NSGA-II iteration is about 10 times slower than an SMS-EMOA iteration.

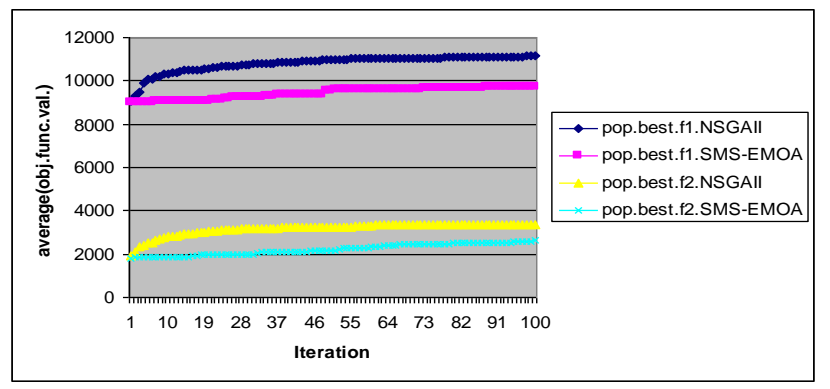

Figure 7: Plots of best values of objective functions (average of 30 runs).

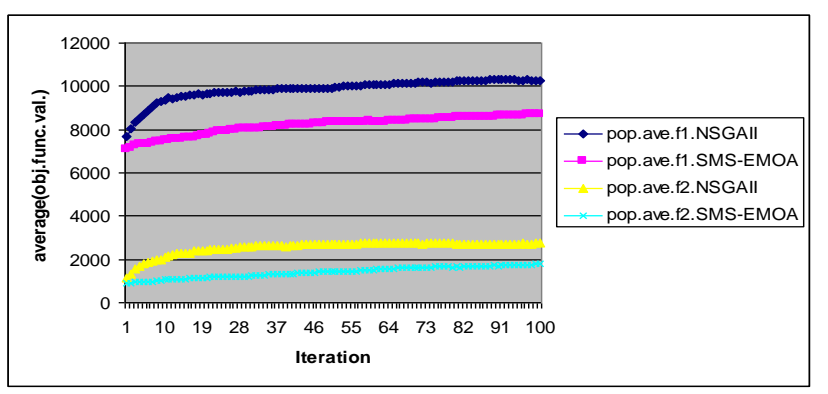

Figure 8: Plots of average values of objective functions (average of 30 runs).

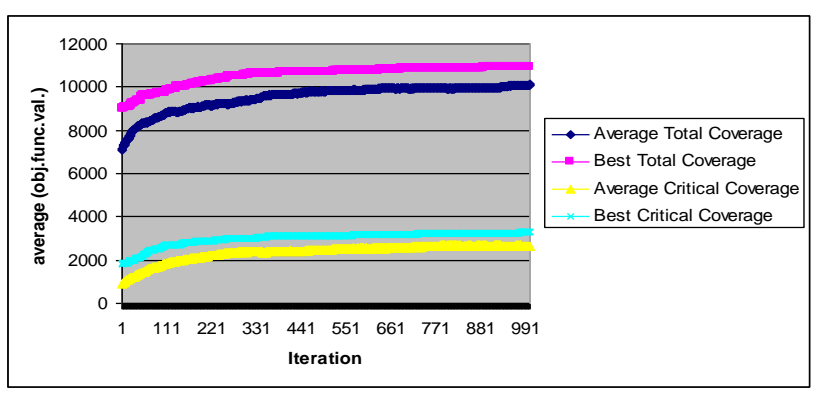

Figure 9: Plots of population best and population average values of 1000 iterations of SMS-EMOA (average of 30 runs).

Our experiments show that the size of the final non-dominated population differs considerably from one run to another for 
both of the approaches. This number is usually smaller for SMS-EMOA approach (between 5 to 11 out of 20 chromosomes) when compared to that of NSGA-II (between 7 to 20 out of 20 chromosomes). The structure of the true Pareto-optimal front may play an important role in this observation.

In order to compare the performances of two approaches, the HI technique, which is discussed in the explanation of SMSEMOA, is applied to measure both convergence and diversity of the obtained non-dominated front in a combined sense [22]. Given a finite search space and a reference point, maximization of the hypervolume measure is equivalent to finding the Pareto-optimal set [23]. It has been also empirically observed that for a fixed number of points, the maximization of the hypervolume metric yields a well distributed subset of the Pareto-optimal front [3]. In our evaluation, the point of zero is taken as the reference for both of the objectives. For normalizing the magnitudes of objective functions, the second objective function value is multiplied by $11792 / 3672$ which is the ratio of the maximum values observed for two objectives. The box plots for HI values are presented in Figure 10. Observing box-plots reveals that, with the applied settings in the experiments, most of the time NSGA-II approach provides more dominated hypervolume according to that of SMS-EMOA.

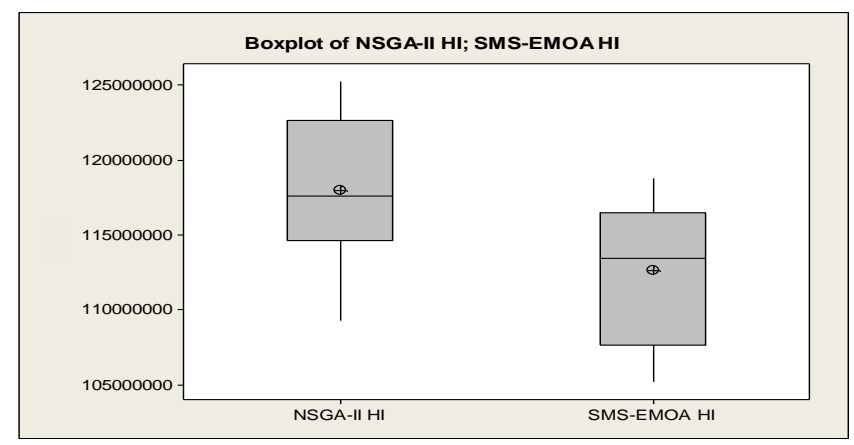

Figure 10: Boxplots for HI values.

The front with the highest dominated hypervolume is seen on Figure 11. In order to create a benchmark, five runs with 500 iterations of NSGA-II are executed and the non-dominated front of the combination of obtained solutions is plotted.

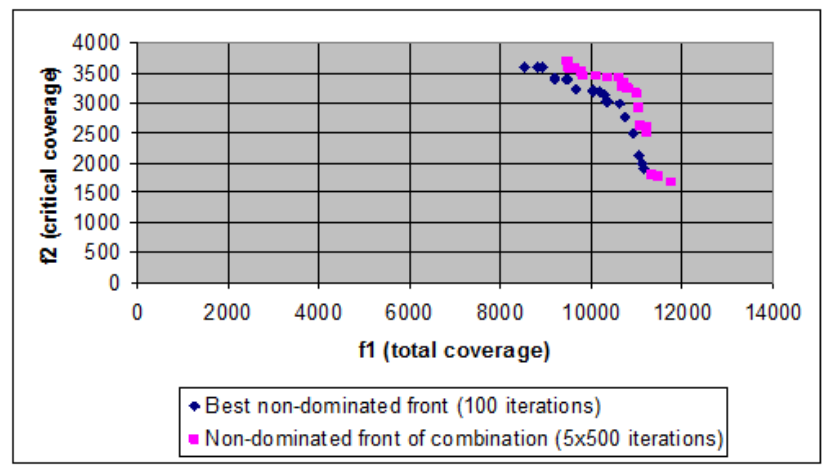

Figure 11: Non-dominated fronts.

\section{Conclusion}

In this study, MOEA methodology is applied to support the decision maker in the problem of stationing radar carrying naval platforms for establishing the most efficient surface picture in the strained period before a possible engagement.
The measure of efficiency has two dimensions, total coverage of radars and the coverage of critical regions.

The application locates the platforms in fleet formation on a digital map of operation area on which critical regions are plotted. As the algorithms evolve, while the set of available escortships is kept fixed, any escortship can change its fleet. In order to approximate the efficient front of the twodimensional solution space, NSGA-II and SMS-EMOA approaches are implemented. The performance of NSGA-II for the experimental problem is found better than that of SMSEMOA in terms of both convergence and diversity. However, while SMS-EMOA evaluates only one offspring at each iteration, NSGA-II calculates objective function values of a number of offspring, as many as parent population, which takes considerably much time.

As a further research, in locating the escortships with respect to a probability distribution within fixed distance limits of the flagship, a local search method can be developed. Local search is expected to improve the solution quality of the approaches presently used in this study, while increasing their running time.

Another improvement idea may be employing a preprocessing step to identify promising regions of the decision space. Moreover, a post-processing step which includes a local search is always applicable after obtaining the final set of nondominated solutions.

\section{References}

[1] Ball MG, Qela B, Wesolkowski S. A Review of the Use of Computational Intelligence in the Design of Military Surveillance Networks. Editors: Abielmona R, Falcon R, Zincir-Heywood N, Abbass HA. Recent Advances in Computational Intelligence in Defense and Security, 663-693, Berlin, Springer, 2016.

[2] Deb K, Pratap A, Agrawal S, Meyarivan T. "A fast and elitist multiobjective genetic algorithm: NSGA-II". IEEE Transactions on Evolutionary Computation, 6(2), 849-858, 2002.

[3] Beume N, Naujoks B, Emmerich M. "SMS-EMOA: multiobjective selection based on dominated hypervolume". European Journal of Operational Research, 181(3), 1653-1669, 2007.

[4] Sakr Z, Wesolkowski S. "Sensor network management using multiobjective evolutionary optimization". IEEE Symposium on Computational Intelligence for Security and Defense Applications (CISDA), Paris, France, 11-15 April 2011.

[5] Oh SC, Tan CH, Kong FW, Tan YS, Ng KH, Ng GW, Tai K. "Multiobjective optimization of sensor network deployment by a genetic algorithm". IEEE Congress on Evolutionary Computation(CEC), Singapore, 25-28 September 2007.

[6] Han JK, Park BS, Choi YS, Park HK. "Genetic approach with a new representation for base station placement in mobile communications". Vehicular Technology Conference, Atlantic City, USA, 07-11 October 2001.

[7] Jiang X, Chen YP, Yu T. "Localized distributed sensor deployment via coevolutionary computation". $3^{\text {rd }}$ International Conference Communications and Networking, Hangzou, China, 13-16 September 2008. 
[8] Fei Z, Li B, Yang S, Xing C, Chen H, Hanzo L. "A Survey of Multi-objective Optimization in Wireless Sensor Networks: Metrics, Algorithms and Open Problems". IEEE Communications Surveys \& Tutorials, 19(1), 550-586, 2016.

[9] Bugajska MD, Schultz AC. "Co-Evolution of form and function in the design of autonomous agents: Micro air vehicle project". Genetic and Evolutionary Computation Conference, Washington DC, USA, 08-12 July 2000.

[10] Bugajska MD, Schultz AC. "Coevolution of form and function in the design of micro air vehicles". NASA/DoD Conference on Evolvable Hardware, Alexandria, VA, USA, USA, 15-18 July 2002.

[11] Chaudhry SB, Hung VC, Guha RK, Stanley KO. "Pareto-based evolutionary computational approach for wireless sensor placement". Engineering Applications of Artificial Intelligence, 24(3), 409-425, 2011.

[12] Martins FV, Carrano EG, Wanner EF, Takahashi RH, Mateus GR. "A hybrid multiobjective evolutionary approach for improving the performance of wireless sensor networks". IEEE Sensors Journal, 11(3), 545-554, 2011.

[13] Khalesian M, Delavar MR. "Wireless sensors deployment optimization using a constrained pareto-based multiobjective evolutionary approach". Engineering Applications of Artificial Intelligence, 53, 126-139, 2016.

[14] Jameii SM, Faez K, Dehghan M. "Multiobjective optimization for topology and coverage control in wireless sensor networks". International Journal of Distributed Sensor Networks, 11(2), 1-11, 2015.

[15] Sengupta S, Das S, Nasir M, Vasilakos AV, Pedrycz W. "An evolutionary multiobjective sleep-scheduling scheme for differentiated coverage in wireless sensor networks". IEEE Transactions on Systems, Man, and Cybernetics, Part C (Applications and Reviews), 42(6), 1093-1102, 2012.
[16] Sengupta S, Das S, Nasir MD, Panigrahi BK. "Multi-objective node deployment in wsns: in search of an optimal trade-off among coverage, lifetime, energy consumption, and connectivity". Engineering Applications of Artificial Intelligence, 26(1), 405-416, 2013.

[17] Bara'a AA, Khalil EA, Cosar A. "Multi-objective evolutionary routing protocol for efficient coverage in mobile sensor networks". Soft Computing, 19(10), 2983-2995, 2015.

[18] Ball MG, Wesolkowski S. "Sensor network placement for maximizing detection of vehicle tracks and minimizing disjoint coverage areas". IEEE International Symposium on Systems Engineerinf (ISSE), Rome, Italy, 28-30 September, 2015.

[19] Küçükali B. Fleet Location and Fuel Consumption Simulation in Naval Combat. MS Thesis, Middle East Technical University, Ankara, Turkey, 2002.

[20] Knowles JD, Corne DW, Fleischer M. "Bounded archiving using the lebesgue measure". Congress on Evolutionary Computation (CEC'03), Canberra, ACT, Australia, 8-12 December 2003.

[21] Knowles J, Corne D. "Properties of an adaptive archiving algorithm for storing nondominated vectors". Evolutionary Computation, IEEE Transactions, 7(2), 100-116, 2003.

[22] Zitzler E, Thiele L. "Multiobjective optimization using evolutionary algorithms-a comparative case study". International Conference on Parallel Problem Solving from Nature, Amsterdam, The Netherlands, 27-30 September 1998.

[23] Fleischer M. "The measure of pareto optima applications to multi-objective metaheuristics". International Conference on Evolutionary Multi-Criterion Optimization, Faro, Portugal, 08-11 April 2003. 